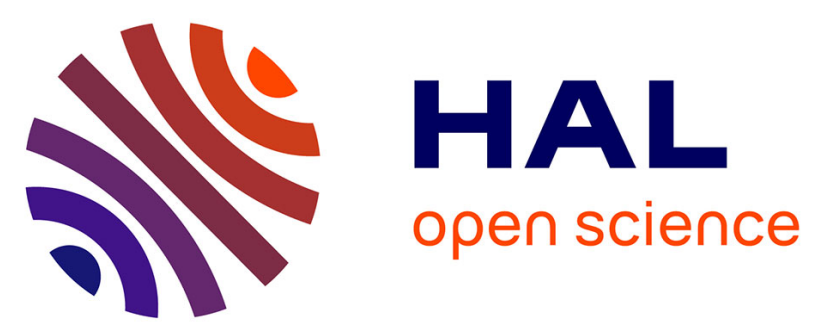

\title{
Effect of Humidity on Dielectric Charging Process in Electrostatic Capacitive RF MEMS Switches Based on Kelvin Probe Force Microscopy Surface Potential Measurements
}

Heiba Zaghloul, G. Papaioannou, Fabio Coccetti, Patrick Pons, Robert Plana

\section{- To cite this version:}

Heiba Zaghloul, G. Papaioannou, Fabio Coccetti, Patrick Pons, Robert Plana. Effect of Humidity on Dielectric Charging Process in Electrostatic Capacitive RF MEMS Switches Based on Kelvin Probe Force Microscopy Surface Potential Measurements. MRS Fall Meeting 2009, Nov 2009, Boston, United States. 6 p. hal-00670181

\section{HAL Id: hal-00670181 https://hal.science/hal-00670181}

Submitted on 14 Feb 2012

HAL is a multi-disciplinary open access archive for the deposit and dissemination of scientific research documents, whether they are published or not. The documents may come from teaching and research institutions in France or abroad, or from public or private research centers.
L'archive ouverte pluridisciplinaire HAL, est destinée au dépôt et à la diffusion de documents scientifiques de niveau recherche, publiés ou non, émanant des établissements d'enseignement et de recherche français ou étrangers, des laboratoires publics ou privés. 


\title{
Effect of Humidity on Dielectric Charging Process in Electrostatic Capacitive RF MEMS Switches Based on Kelvin Probe Force Microscopy Surface Potential Measurements
}

\author{
U. Zaghloul $^{1,2}$, G.J. Papaioannou ${ }^{1,3}$, F. Coccetti ${ }^{1,4}$, P. Pons ${ }^{1}$ and R. Plana ${ }^{1,2}$ \\ ${ }^{1}$ CNRS ; LAAS ; 7 avenue du colonel Roche, F-31077 Toulouse, France \\ ${ }^{2}$ Université de Toulouse ; UPS, INSA, INP, ISAE ; LAAS ; F-31077 Toulouse, France \\ ${ }^{3}$ University of Athens, Solid State Physics, Panepistimiopolis Zografos, 15784 Athens, Greece \\ ${ }^{4}$ Novamems, 10 av. De l'Europe, F-31520 Toulouse, France
}

\begin{abstract}
In this article we investigate the effect of relative humidity on dielectric charging/discharging processes in electrostatically actuated MEMS devices. The assessment procedure is based on surface potential measurements using Kelvin Probe Force Microscopy (KPFM) and it targets in this specific work PECVD silicon nitride films in view of application in electrostatic capacitive RF MEMS switches. Charges have been injected through the AFM tip and the induced surface potential has been measured under different relative humidity levels. The impact of the charge injection duration and the bias level as well as bias polarity applied during the charge injection step, have been explored. Finally, the effect of the dielectric film thickness under different relative humidity levels has been assessed through depositing SiN films with different thicknesses.
\end{abstract}

\section{KEYWORDS}

Dielectric charging, Humidity, Silicon nitride, Kelvin Probe Force Microscopy

\section{INTRODUCTION}

A very few work has been performed in order to study the effect of humidity on the dielectric charging process in RF-MEMS switches and therefore a full understanding of this effect is still missing. Surface charging has been reported to be more detrimental to switch lifetime than bulk charging [1]. Besides, based on measuring the shift in the switch actuation voltage, bulk charging was found to dominate in dry air, while surface charging was reported to increase linearly with increasing humidity [2]. Finally, it is reported that under comparable humidity levels and electric fields, switches employing silicon dioxide dielectric are less susceptible to surface charging than switches employing silicon nitride [1].

Recently, Kelvin Probe Force Microscopy (KPFM) has proven to be a very efficient method in assessing the dielectric charging process [3-7]. For example, the surface potential distribution was found to be more confined in thinner dielectric films when the same bias is applied during charge injection, independently on the substrate nature [6]. Yet, all previous work done up to now which made use of the KPFM for dielectric charging assessment has been performed in ambient air and hence the effect of humidity and the surface charge have not been addressed. On the other sides, the removal of the water-related layer from both surfaces on the sample and the AFM tip is reported to be very important to improve the reliability of the KPFM measurements [8]. At low humidity $\approx 10 \% \mathrm{RH}$ trapped surface charges could be detected in the KPFM measurements while such charging effects become less prominent at high humidity $\approx 80 \% \mathrm{RH}[9]$. Finally, the adsorbed water layer on the sample surface is considered to shield the surface potential [10]

This work therefore presents the effect of humidity and in sequence the surface charge on the charging/discharging processes in PECVD silicon nitride films based on KPFM methodology. The KPFM measurements have been performed under different relative humidity levels. Charges have been injected using different bias levels and various injection durations for both positive and negative bias 
polarities. Finally, the effect of the dielectric film thickness under different relative humidity levels has been investigated.

\section{KPFM EXPERIMENTAL SETUP}

In our KPFM experiments, charges are first injected in single points over the SiNx surface by applying voltage pulses of defined amplitude, Up, and duration, Tp, to the AFM tip (Fig 1.a). Then the surface potential decay is measured with time. The analysis of the results is performed by measuring the full width at half maximum (FWHM) as well as the surface potential peak directly after charge
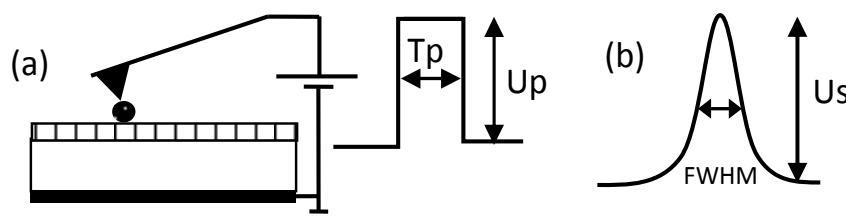

Figure 1. The procedure of the KPFM experiment and result analysis (a) charge injection in single point, (b) resulting surface potential distribution. injection for each injected point charge (Fig. 1.b). The effect of the charge injection duration, $\mathrm{Tp}$, has been investigated through injecting charges for different durations in the range from $0.01 \mathrm{sec}$ to $100 \mathrm{sec}$. Besides, the impact of the applied bias amplitude, Up, has been explored through applying Up pulses of $10 \mathrm{~V}$ and $20 \mathrm{~V}$ amplitude. Finally, the experiments have been performed for both positive and negative Up.

The surface potential measurements have been performed under two different relative humidity levels; in ambient air $(\mathrm{RH} \approx 40 \%)$ and under dry air flow $(\mathrm{RH} \approx 6 \%)$. The samples which have been measured under dry air flow have been first dehydrated at $120^{\circ} \mathrm{C}$ for two cycles during three hours under vacuum just before the KPFM measurements. The SiN films were deposited by high frequency (HF) power supply (13.56 MHz) PECVD method over a Ti/Au layers which have been evaporated over low resistivity silicon substrate. In order to investigate the effect of dielectric thickness, two different film thicknesses have been investigated; $200 \mathrm{~nm}$ and $300 \mathrm{~nm}$.

The KPFM measurements have been performed using the Digital Instrument Dimension-3100 equipped with Nanoscope IV controller. SCM-PIT conductive AFM tips from Veeco, which are Antimony n-doped silicon and coated with platinum/iridium, have been used. Here it should be mentioned that the measured surface potential varies from one tip to another due to the differences in geometry and intrinsic characteristics of each tip. Therefore, the same tip has been used for all measurements in order to be able to compare the results for different samples precisely. The employed tip-sample separation in our measurements was $20 \mathrm{~nm}$. Moreover, the parameters used during the KPFM measurements were optimized using the scheme presented in [9]. Finally, the KPFM experiments have been performed several times for each sample under different charge injection conditions and the error percentage in the results presented in this work is kept less than $5 \%$.

\section{RESULTS AND DISCUSSION}

Charges have been injected through the AFM tip in both contact and tapping modes. The impact of the relative humidity levels on the surface potential decay as well as on the lateral charge spreading for charges which have been injected in contact mode will be addressed in the following section. Then, the effect of the humidity on the surface potential distribution will be investigated for charges that have been injected in tapping mode for different duration and under different bias amplitudes.

\section{Effect of humidity on surface potential decay and redistribution}

For this set of experiments, charges have been injected in contact mode using a pulse amplitude, $\mathrm{Up}$, of $10 \mathrm{~V}$ and a pulse duration, Tp, of $60 \mathrm{sec}$ and the measurements have been performed for both the 
200nm and 300nm SiN films. Figure 2(a, b) presents a comparison between the time dependence of the surface potential measured under different relative humidity levels, for both investigated SiN samples. For both investigated SiN thicknesses and under both $6 \%$ and $40 \%$ relative humidity levels, the surface potential is found to decay exponentially with time following the stretched exponential law, $\exp \left[-\left(\frac{t}{\tau}\right)^{\beta}\right]$, where $\tau$ is the process time constant and $\beta(0 \leq \beta \leq 1)$ is the stretch factor [7]. As can be observed, the decay time constant in case of measurements under dry air is much longer than in case of measuring the surface potential in ambient air. In case of $6 \% \mathrm{RH}$, a KPFM signal of around $73 \%$ and $58 \%$ of the initial potential at $\mathrm{t}=0$ for the $200 \mathrm{~nm}$ and $300 \mathrm{~nm}$ samples respectively, could be detected even after more than 11 hours of measurements. On the contrary, in case of measurements performed under $40 \% \mathrm{RH}$, the measured KPFM potential is around $17 \%$ and $13 \%$ of its initial potential at $\mathrm{t}=0$ for the $200 \mathrm{~nm}$ and $300 \mathrm{~nm}$ samples respectively, after 35 minutes only.
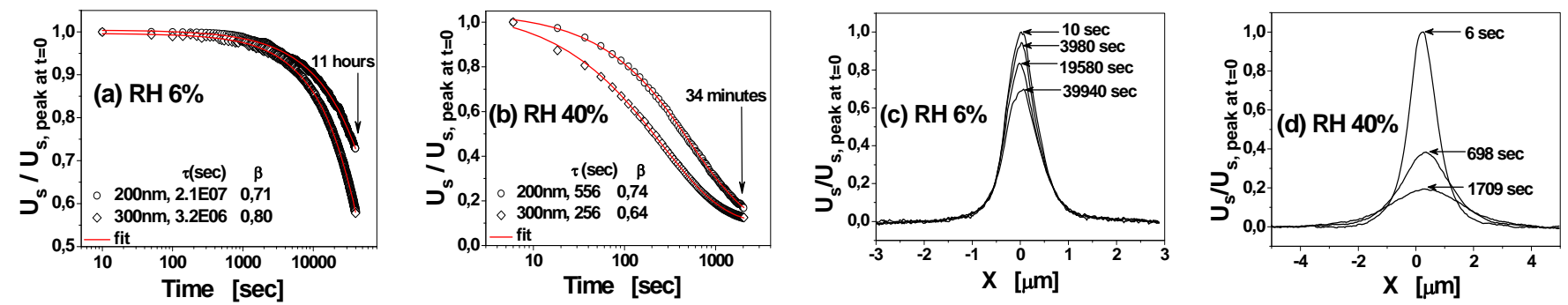

Figure 2. Impact of relative humidity on surface potential decay and redistribution with time. Surface potential decay for $\mathrm{SiN}$ films of $200 \mathrm{~nm}$ and 300nm thickness at (a) $6 \% \mathrm{RH}$ and (b) $40 \% \mathrm{RH}$. Redistribution of the surface potential with time for the $200 \mathrm{~nm} \mathrm{SiN}$ film at (c) $6 \% \mathrm{RH}$ and (d) $40 \% \mathrm{RH}$.

There are several reasons of possible charge decay, which are usually classified into two groups: external and internal charge decay. The external decay is due to the neutralisation of surface charges, which can be caused by the adsorption of counterions from the surrounding medium or by charge loss due to its electrical conductivity. In the case of air as environment the amount of available counterions and the electrical conductivity are both influenced by the humidity. On the other side, the internal decay is caused by the small but non-zero electrical conductivity of the dielectric material and by diffusion of charge carriers. Diffusion plays mostly a minor role in charge decay [11]. As surface charges are predominantly subjected to external charge decay and volume charges are predominantly subjected to internal decay which is not affected by a change of the environmental conditions, the huge observed difference in the decay time constant between measurements under $6 \%$ and $40 \% \mathrm{RH}$ is attributed to the excess surface charge decay in case of higher relative humidity levels due to a possible thin water film over the dielectric surface. This water film has been considerably removed during the dehydration step under vacuum performed for the SiN samples measured under dry air flow.

Comparing the values of the decay time constants for the $200 \mathrm{~nm}$ and $300 \mathrm{~nm}$ SiN films for KPFM measurements performed under dry air (Fig. 2a) and in ambient air (Fig. 2b), it can be observed that the time constant is larger in the thinner dielectric films than in the thicker ones [7], independently of the employed relative humidity. The longer time constants in case of thin dielectric films can be attributed to the fact that the thinner material will be affected more by the defective first deposited SiN layers. Such layers will contain more complex defects with large capture cross section located deep in the band gap. In the case of the thick dielectric film on the contrary the top deposited layers are expected not to be affected by these disordered bottom layers. In other words, the defects in these top layers are expected to exhibit simpler structure and have smaller capture cross section.

On the other side, the lateral charge redistribution of the injected charges has been investigated 
through monitoring the evolution of the potential distribution with time, Fig. 2 (c, d). No lateral charge spreading has been observed when the KPFM experiments have been performed under dry air with $6 \%$ RH even after 11 hours (Fig. 2c). On the contrary, lateral charge redistribution is clearly observed after 35 minutes only in case of surface potential measurements performed in ambient air with $40 \% \mathrm{RH}$ (Fig. 2d), in spite of the low reported mobility of electrons in SiN [4]. This can be attributed to two main reasons. First, the dehydration step which has been performed for the SiN samples measured under dry air flow. Such annealing and cooling steps under vacuum are expected to remove a considerable part of the water film which already exists over the dielectric surface. Second, the KPFM measurements under dry air will prevent the water condensation over the dielectric surface. These two reasons will thereby inhibit lateral charge migration due to the electrical conductivity of the mentioned possible water film [12].

Here it should be pointed out that the evolution of the surface potential from KPFM measurements in ambient air showed that the charges practically do not diffuse across the surface during charge dissipation and the decay of the charge was attributed to transport and trapping into the bulk of the dielectric $[3,4]$. Besides, it should be emphasized that although lateral charge redistribution is observed in our KPFM measurements performed in ambient air $(40 \% \mathrm{RH})$, this cannot justify the previously observed surface potential fast decay (Fig. 2b). Therefore, the relation between the lateral charge spreading and the surface potential decay is still under further investigation.

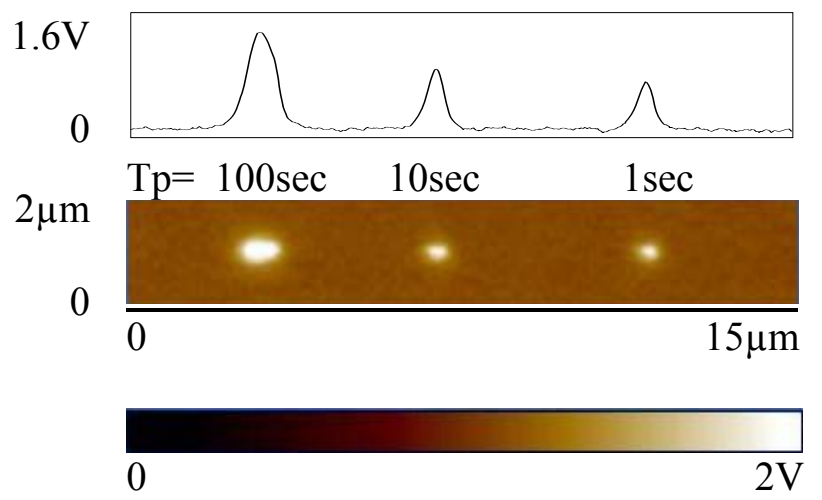

(a) RH 6\%

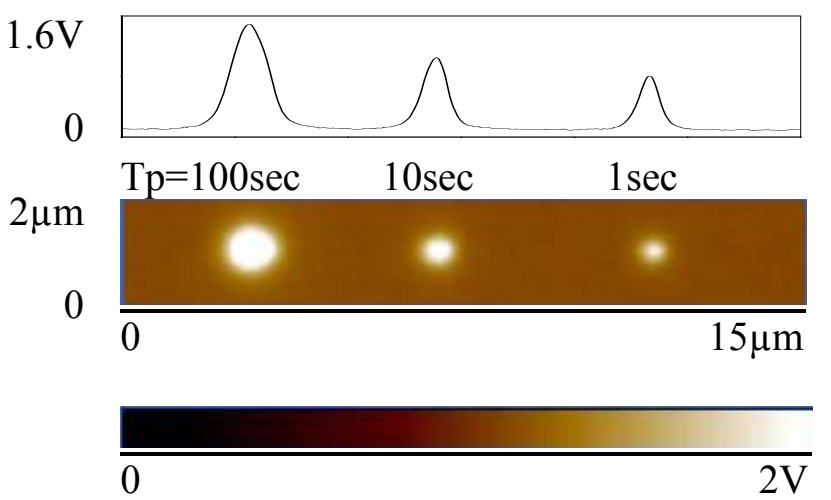

(b) $\mathrm{RH} 40 \%$

Figure 3. The measured surface potential at (a) $6 \% \mathrm{RH}$ and (b) $40 \% \mathrm{RH}$ for charges injected in three different positions each for different charge injection time, Tp, $(100 \mathrm{sec}, 10 \mathrm{sec}$ and $1 \mathrm{sec}$ from left to right respectively), pulse amplitude, $\mathrm{Up}$, is $20 \mathrm{~V}$ and the SiN film thickness is $200 \mathrm{~nm}$.

\section{Effect of humidity on surface potential distribution}

The results presented in this section are for charges which have been injected in tapping mode for different duration, Tp, different bias amplitude, $\mathrm{Up}$, and with both positive and negative bias polarities (see Fig. 1). The investigated time range for Tp is from $0.01 \mathrm{sec}$ to $100 \mathrm{sec}$ while two different bias amplitudes have been used; $10 \mathrm{~V}$ and $20 \mathrm{~V}$. The FWHM and hence the surface potential distribution resulting from charges which have been injected in different points, are measured directly after the charge injection step. Figure 3 presents a sample of these results where a row of single point charges separated by $4 \mu \mathrm{m}$ each has been injected and measured under dry air flow (Fig. 3a) and in ambient air (Fig. 3b) using different pulse duration, Tp, and under pulse amplitude of 20V. The charge injection in different points has been performed using the NanoScript software available from Veeco, which controls the tip movements on the sample surface during the charge injection step. It is clearly observed from the figure that both the FWHM as well as the surface potential amplitude, Us, increase remarkably with increasing the pulse duration. 
Figure 4 shows the FWHM versus the charge injection duration ,Tp, for the $200 \mathrm{~nm}$ SiN sample measured under dry air (6\% RH) and in ambient air (40\%) for charges which have been injected for different pulse amplitude levels $(10 \mathrm{~V}$ and $20 \mathrm{~V})$ with both positive and negative bias polarities. It is clear from the figure that the FWHM increases with increasing the charge injection duration, Tp. This is found to apply for both $10 \mathrm{~V}$ and $20 \mathrm{~V}$ bias amplitudes, positive and negative bias polarities, and finally for measurements under both $6 \%$ and $40 \% \mathrm{RH}$ levels. Besides, from the comparison between the FWHM for Up of $20 \mathrm{~V}$ and $10 \mathrm{~V}$, at either $6 \%$ or $40 \% \mathrm{RH}$ levels (Fig. $4 \mathrm{a}$ ), it is noticed that as the applied Up increases, the FWHM gets wider and hence the surface potential distributions becomes less confined.

Concerning the effect of relative humidity on the surface potential distribution, Fig. 4a highlights that the FWHM is smaller in case of KPFM measurements performed under $6 \% \mathrm{RH}$ than in experiments performed in $40 \% \mathrm{RH}$ for both Up of $20 \mathrm{~V}$ and $10 \mathrm{~V}$. In other words the measured surface potential becomes more confined as the relative humidity decreases. Moreover, the rate of increase of FWHM with the injection time is found to be more pronounced with higher bias at higher relative humidity levels. These observations have been found to apply as well when using negative charge injection, Fig. 4b. The impact of relative humidity on the FWHM and hence on the surface potential distribution can be directly related to the difference in the conductivity of the possible water film and hence the amount of the surface charge which exists on the dielectric surface as the relative humidity changes. Basically, the measured surface potential distribution follows the electric field distribution which exits during the charge injection step between the AFM tip and the SiN sample ground plane. As reported in [13], the distribution of the electric field just under the probe becomes less concentrated as the adsorbed water increases. Hence, as the conductivity as well as the amount of surface charge are expected to be smaller for the KPFM measurements performed at the $6 \% \mathrm{RH}$ due to the reasons explained earlier, the electric field distribution and consequently the induced surface potential are expected to be more confined comparing to the KPFM measurements occurred in ambient air. Another contribution could be attributed to the expansion of the apparent tip curvature radius by the adsorbed water layer at higher relative humidity levels [10].

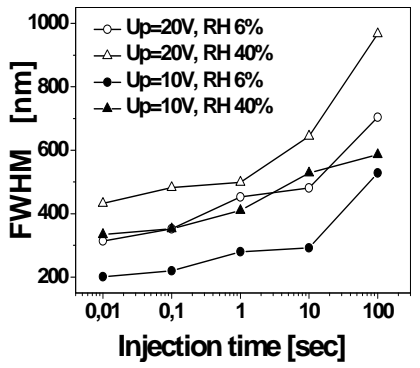

(a)

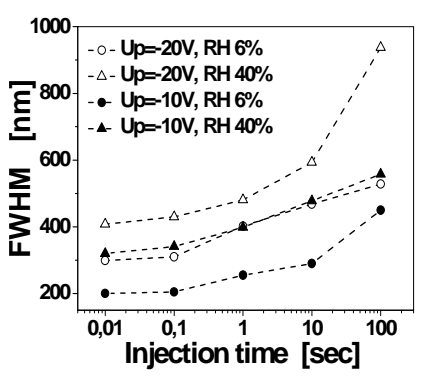

(b)

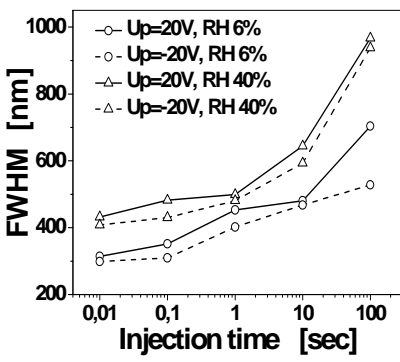

(c)

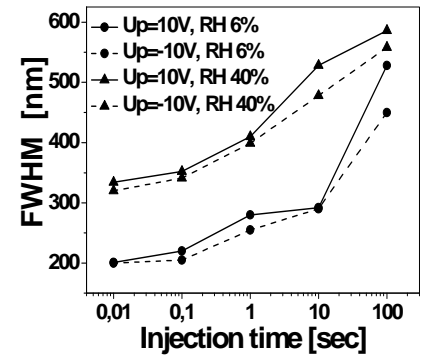

(d)

Figure 4. The measured FWHM under $6 \%$ RH and $40 \%$ for the $200 \mathrm{~nm}$ SiN sample, versus the charge injection time, $\mathrm{Tp}$, for (a) different positive pulse amplitude, $10 \mathrm{~V}, 20 \mathrm{~V}$ (b) different negative pulse amplitude, $-10 \mathrm{~V},-20 \mathrm{~V}$, (c) different bias polarities $20 \mathrm{~V},-20 \mathrm{~V}$, (d) different bias polarities, $10 \mathrm{~V},-10 \mathrm{~V}$.

Comparing the FWHM values when positive or negative bias Up are applied to the AFM tip reveals that the surface potential distribution resulting from the negative charge injection is more confined than in case of applying positive bias, Fig. 4(c, d). Moreover, this has been observed for both the investigated bias levels, 20V (Fid. 5c) and 10V (Fig. 4d). This can be attributed to an asymmetric charging behavior which have been observed in both symmetric [14] and asymmetric [15] MIM capacitors. Moreover, comparing Fig. 4c and Fig. 4d reveals that the difference between the FWHM measured in $6 \% \mathrm{RH}$ and $40 \%$, is more pronounced as the applied bias gets smaller. As discussed earlier the FWHM increases as the applied bias, Up, increases. Therefore, for the 20V applied bias the FWHM 
is expected to be already large and then the charge spreading can be fairly enhanced by the relative humidity variation. While in case of the $10 \mathrm{~V}$ bias, the FWHM is already small enough to be affected sharply by the variation in the relative humidity levels. In other words, the effect of changing the relative humidity on the FWHM is not very obvious being overwhelmed by the impact of the high bias applied.

\section{CONCLUSION}

The effect of the relative humidity on the dielectric charging process in PECVD silicon nitride films has been investigated based on the surface potential measurements from Kelvin Probe Force Microscopy. Two different humidity levels have been investigated; $6 \%$ under dry air flow and $40 \%$ in ambient air. Various parameters have been investigated which are the dielectric film thickness, charge injection duration and charge injection bias amplitude and polarity. The investigation reveals that the potential distribution was found to be more confined as the relative humidity decreases especially at smaller charge injection bias. The possible water film as well as the excess surface charge which exists at high relative humidity levels is found to contribute to the fast charge decay as well as the lateral charge redistribution. The surface potential was found to decay following the stretched exponential low for KPFM experiments performed under the two investigated humidity levels. Moreover, thinner dielectric films were found to have longer time constants, independently of the employed relative humidity level. Finally, asymmetry has been observed when positive and negative bias is applied during the charge injection.

\section{ACKNOWLEDGEMENTS}

This work has been partially supported by the following projects: the French ANR project FAME (PNANO-059), POLYNOE project funded by European Defense Agency (B-0035-IAP1-ERG), and the SYMIAE project funded by the Fondation STAE.

\section{REFERENCES}

[1] Z. Peng et al., IEEE Microwave and Wireless Components Letter, 19, no5, pp. 299-301(2009).

[2] Z. Peng et al., Proc. Asia-Pacific Microwave Conference, pp. 1535-1539, (2006).

[3] L. Lamhamdi et al., the $14^{\text {th }}$ International Conference on Solid-State Sensors, Actuators and Microsystems, Lyon, France, pp 579-582, (2007).

[4] R. W. Herfst et al., the $46^{\text {th }}$ Annual International Reliability Physics Symposium, Phoenix, pp. $492-$ 495, (2008).

[5] A. Belarni et al., Journal of Microelectronics Reliability 48, pp. 1232-1236, (2008).

[6] U. Zaghloul et al., MRS Fall Meeting, Boston, DOI: 10.1557/PROC-1139-GG03-37, (2008).

[7] U. Zaghloul et al., the $15^{\text {th }}$ International Conference on Solid-State Sensors, Actuators, and Microsystems, (Transducers) Denver, pp. 789-793, (2009).

[8] S. Ono, M. Takeuchi, and T. Takahashi, Appl. Phys. Lett. 78, 1086, (2001).

[9] H. O. Jacobs, H. F. Knapp, A. Stemmer, Rev. Sci. Instrum. 70, pp. 1756- 1760, (1999).

[10] H. Sugimura, Y. Ishida, K. Hayashi O. Takai, and N. Nakagiri, Appl. Phys. Lett. 80, 1459, (2002).

[11] G.M. Sessler (Ed.), Electrets, "Springer topics in applied physics", vol. 33, Springer Verlag, Berlin, 2nd ed., (1987).

[12] H.F. Knapp, A. Stemmer, Surf Interface Anal 27 (5-6): 324-331, (1999).

[13] K. Ohara and Y. Cho, J. Appl. Phys. 96, 7460, (2004).

[14] K. J. B. M. Nieuwesteeg et al., J. Appl. Phys., 71, pp. 1290-1297, (1992).

[15] U. Zaghloul et al., Journal of Microelectronics Reliability, 49, pp. 1309-131(2009). 